TITLE:

\title{
Magnetism of fcc/fcc, hcp/hcp twin and fcc/hcp twin-like boundaries in cobalt
}

\section{AUTHOR(S):}

Hakamada, Masataka; Hirashima, Fumi; Kajikawa, Kouta; Mabuchi, Mamoru

\section{CITATION:}

Hakamada, Masataka ... [et al]. Magnetism of fcc/fcc, hcp/hcp twin and fcc/hcp twin-like boundaries in cobalt. Applied Physics A: Materials Science and Processing 2012, 106(1): 237-244

\section{ISSUE DATE:}

2012-01

URL:

http://hdl.handle.net/2433/237665

\section{RIGHT:}

This is a post-peer-review, pre-copyedit version of an article published in 'Applied Physics A: Materials Science and Processing'. The final authenticated version is available online at: https://doi.org/10.1007/s00339-011-6568-9./ The fulltext file will be made open to the public on 11 September 2012 in accordance with publisher's 'Terms and Conditions for Self-Archiving'.: This is not the published version. Please cite only the published version.; この論文は出版社版であり ません。引用の際には出版社版をご確認じ利用ください。 


\section{Magnetism of $f c c / f c c, h c p / h c p$ twin and $f c c / h c p$ twin-like boundaries in cobalt}

Masataka Hakamada $^{1}$, Fumi Hirashima ${ }^{1}$, Kota Kajikawa ${ }^{1}$ and Mamoru Mabuchi ${ }^{1}$

1 Department of Energy Science and Technology, Graduate School of Energy Science, Kyoto

University, Yoshidahonmachi, Sakyo, Kyoto 606-8501, Japan

Corresponding author: Masataka Hakamada

Tel.: +81 75753 5427; fax: +81 757535428 .

E-mail address: hakamada.masataka.3x@kyoto-u.ac.jp 


\section{Abstract}

The magnetic moments of the $f c c / f c c, h c p / h c p$ twin and $f c c / h c p$ twin-like boundaries in cobalt were investigated by first-principles calculations based on density functional theory. The magnetic moments in $f c c / f c c$ were larger than those of the bulk $f c c$, while the variations in the magnetic moment were complicated in $h c p / h c p$ and $f c c / h c p$. The magnetovolume effect on the magnetic moment at the twin(-like) boundaries was investigated in terms of the local average atomic distance and the average deviation from equilibrium; however, the complicated variations in the magnetic moment could not be explained from the magnetovolume effect. Next, the narrowing (or broadening) of the partial density of states (PDOS) width of $3 d$ orbitals, the number of occupied states for the spin-down channel and the PDOS around the Fermi level were investigated. The entire variation in the magnetic moment at the twin(-like) boundaries could be understood in terms of these factors. Charge transfer occurred in $h c p / h c p$. In this case, the contributions of $4 s$ and $4 p$ electrons to the variation in the magnetic moment were relatively large. 


\section{Introduction}

The magnetic moment of an atom can be interpreted from the viewpoint of the shape and width of the density of states (DOS): the magnetic moment is often enhanced by narrowing the DOS width of the $d$ bands or by increasing the DOS around the Fermi level. The shape and width of the DOS change at planar defects such as grain boundaries (GB) and free surfaces, resulting in an enhancement or a reduction in the magnetic moment at planar defects [1-6]. Hampel et al. [1] suggested that the bonding at the GB is inhibited among the directional $d$ orbitals owing to changes in the coordination number and coordination geometry and that the width of the $d$ band is narrowed; as a result, the magnetic moment is enhanced at the GB. To date, an enhancement of the magnetic moment at the GB has been observed in iron [1-5] and nickel [3] by first-principles calculations. These studies pointed out the importance of the narrowing of the $d$ band width for enhancing the magnetic moment at the GB. Recently, the enhanced magnetic moment at the GB has been demonstrated experimentally [7], where the magnetic moment at the GB was more than two times larger than that of the bulk. Also, it was found that nanocrystalline Fe exhibited a large saturation magnetization [8]. On the other hand, a reduction in the magnetic moment due to the presence of GB has been observed experimentally (with a vibrating sample magnetometer) [9] and numerically (with first-principles simulation) [10]. Thus, the magnetic moment at the GB is still in debate. The discrepancy in the magnetic moment variation at the GB may be because the grain boundary characteristics such as the coordination number and coordination geometry are too complex to be 
determined with accuracy.

Twin boundaries are GB with special symmetry and low disorder. Because the coordination number of atoms at twin boundaries is the same as that in the bulk, only the coordination geometry affects the magnetic moment of twin boundaries. The effects of twin boundaries on magnetisms have been investigated in many studies [11-14]. Sampedro et al. [13] revealed that ferromagnetism of Pd nanoparticles is due to their twin boundaries. Also, Alexandre et al. [15] showed that the magnetic susceptibility is enhanced at twin boundaries. Recently, it has been reported that nanocrystalline Co having a nanoscale lamellar structure with a spacing of $3 \mathrm{~nm}$ exhibited larger saturation magnetization than bulk hcp Co [16], where the nanolamellar structure consisted of twins. The variation in the magnetic moment is limited to a few layers adjacent to the GB [1]. Hence, twins can affect the saturation magnetization when the spacing between twin boundaries is of nanometer order. Co nanotwins consist of $f c c$ and $h c p$ phases [17] because the stable phase of Co depends on not only the temperature but also the size [18]. In the present work, the magnetic moments of $f c c / f c c, h c p / h c p$ nanotwin and $f c c / h c p$ nanotwin-like structures in cobalt are investigated by first-principles calculations based on density functional theory (DFT) using the CASTEP code [19]. The variations in the magnetic moments at the twin(-like) boundaries are analyzed from the viewpoint of the magnetovolume effect and the shape and width of the $d$ bands.

\section{Simulation methods}


Four models of the $f c c / f c c, h c p / h c p(1), h c p / h c p(2)$ twin and $f c c / h c p$ twin-like boundaries in cobalt were used (Fig. 1). The stacking sequence was ABCBACABCBAC for the $f c c / f c c$ model and ABCABABABCABAB for the $f_{c c} / h c p$ model along the [111] direction. The twin planes of the hcp/hcp(1),(2) models were $(10 \overline{1} 1) /(10 \overline{1} 1)$ and $(11 \overline{2} 4) /(11 \overline{2} 4)$, respectively. The fcc/hcp can be regarded as an $h c p$ twin, where a unit of CA stacking is the symmetry plane. The $f c c / f c c, h c p / h c p(1)$, hcp/hcp(2) and fcc/hcp models have 6, 52, 36, 7 atoms of Co in one supercell, respectively. The calculations of magnetic moments were performed using the CASTEP code, where DFT [20,21] was used to calculate the electronic properties of the four models. Infinite lattice systems were used with periodic boundary conditions. The models were, therefore, ideal for calculations of periodic systems. The exchange-correlation interactions were treated using the spin-polarized version of the generalized gradient approximation (GGA) within the scheme proposed by Perdew-Burke-Ernzerhof (PBE) [22]. The valence electrons described by Vanderbilt-type nonlocal ultrasoft pseudopotentials were Co $3 d^{7} 4 s^{2}$. Ultrasoft pseudopotentials [23] represented in reciprocal space were used for all elements in our calculations. All atomic positions were optimized with respect to all structural parameters in accordance with Hellman-Feynman forces and stresses using the Broyden-Fletcher-Goldfarb-Shanno (BFGS) algorithm [24]. The optimization calculations were performed until the convergence criteria were satisfied, that is, $5.0 \times 10^{-6} \mathrm{eV}$ for the energy change per atom, $0.01 \mathrm{eV} \AA^{-1}$ for the RMS force, 0.02 GPa for the RMS stress and $5.0 \times 10^{-4} \AA$ for the RMS displacement. The cutoff energy was set at $330 \mathrm{eV}$ for all models. The Brillouin zone was sampled 
with the Monkhorst-Pack $k$-point grid. A $16 \times 16 \times 3 k$-point mesh was used for the $f c c / f c c$, a $3 \times 10 \times 1$ $k$-point mesh for the $h c p / h c p(1)$, a $6 \times 4 \times 2 k$-point mesh was used for the $h c p / h c p(2)$ and a $16 \times 16 \times 3$ $k$-point mesh was used for the $f c c / h c p$. Mulliken populations were employed to obtain the magnetic moment per atom. All the calculations of the lattice parameters and magnetic moments were performed after optimization calculations for the most stable geometry. The lattice parameters of bulk $f c c$ and hcp Co were calculated by optimization calculation; as a result, the lattice parameter was $3.513 \AA$ for the bulk $f c c$ Co and $2.520 \AA$ (a-axis) and $4.069 \AA$ (c-axis) for bulk hcp Co. These values are almost the same as those in the previous work [25]. The magnetic moment of an atom in bulk Co was $1.68 \mu \mathrm{b}$ for the $f c c$ phase and $1.68 \mu \mathrm{b}$ for the hcp phase. In addition, the magnetic moments of bulk Co were calculated under the condition that the lattice parameter was forced to be isotropically expanded or shrunk to investigate the magnetovolume effect. The individual contributions of $3 d$, $4 s$ and $4 p$ electrons to the magnetic moment were obtained from the difference between the spin-up and spin-down electrons by integrating the partial DOS within each atomic sphere. In the present work, all the calculations were performed at $0 \mathrm{~K}$. Recently, Polesya et al. [26] investigated the temperature dependence of the average magnetic moment of free Fe clusters consisting of 9-89 atoms and they showed that an average magnetic moment at about $300 \mathrm{~K}$ is essentially the same as that at $0 \mathrm{~K}$. This suggests that the temperature does not affect the theoretical results for the magnetic properties in the range of $0-300 \mathrm{~K}$.

Also, effects of the twin spacing in the $f c c / f_{c c}$ model (Fig. 1 (a)) were investigated using the 
modified $f c c / f c c$ model with a 6-atomic-layer cell. As a result, the similar results were obtained for both the 6-atomic-layer cell model and the 3-atomic-layer cell model. Thus, effect of the twin spacing on the magnetic moment was minor.

\section{RESULTS AND DISCUSSION}

\subsection{Magnetic moments}

The magnetic moments of the Co atoms for the $f c c / f c c, h c p / h c p(1), h c p / h c p(2)$ and $f c c / h c p$ are listed in Table 1 . In the $f c c / f c c$, the magnetic moments were larger than those of the bulk $f c c$, and were independent of the location, at least under the conditions investigated. On the other hand, the magnetic moments in the $h c p / h c p(1)$, except for Co1 and Co7, were less than those of the bulk hcp, while the magnetic moment of Co1 was equal to that of the bulk hcp and that of Co7 was much larger. In the $h c p / h c p(2)$, the magnetic moments of Co2, Co3, Co5, Co6, Co8 and Co9 were equal to those of the bulk hcp, but the magnetic moment of Co1 was larger than that of the bulk hcp and the magnetic moments of $\mathrm{Co} 4$ and Co7 were much lower. As a whole, the magnetic moment tended to be reduced at the $h c p$ twin boundaries with a few exceptions, such as the greatly enhanced moment of Co7 in the $h c p / h c p(1)$, while it was enhanced at the $f c c$ twin boundaries. These trends were also found in the fcc/hcp.

The magnetic moment is changed by about $15 \%$ in the case where the boundary structure is fully disordered [10]. In the present work, the magnetic moment was changed by at most only $7 \%$, as 
shown in Table 1 . This is reasonable, considering the intensity of structural disorder of twins. On the other hand, the variation in the magnetic moment was not monotonic for the twins. A complicated variation in the magnetic moment was obtained for the $\Sigma 5(210) \mathrm{GB}$ in iron; however, it was attributed to the variation in the coordination number $[2,4]$. Clearly, the complicated variations in the magnetic moment at the twin(-like) boundaries are related to local disorder or reconstruction [15].

Table 2 shows the individual contributions of $3 d, 4 s$ and $4 p$ electrons to the magnetic moments for the $f c c / f c c, h c p / h c p(1), h c p / h c p(2), f c c / h c p$, bulk $f c c$ and bulk hcp in cobalt. It can be seen that the $3 d$ electrons dominantly contribute to the magnetic moments. The roles of $4 s$ and $4 p$ electrons will be discussed later.

\subsection{Effects of atomic distance}

The magnetic moment at planar defects is affected by changes in the coordination number and coordination geometry. However, because the coordination number of atoms at twin boundaries is the same as that in the bulk, the variations in the magnetic moment in Table 1 are related to the coordination geometry. It has been demonstrated by many experiments with SQUID magnetometer [27] and x-ray magnetic circular dichroism [28], and first-principles simulations [3,29,30] that magnetovolume or magnetostriction affects the magnetic moment.

Szpunar et al. [10] used the local average atomic distance and the average deviation from equilibrium to quantify the geometry of each atom, where the local average atomic distance is 
defined as the average distance between a given site of an atom and its nearest neighbors, and the average deviation from equilibrium is defined as the average deviation from the nearest-neighbor distance. The relationship between the magnetic moment and local average atomic distance is shown in Fig. 2 (a), while a plot of the magnetic moment $v s$ the average deviation from equilibrium is shown in Fig. 2 (b), for atoms in the four twin(-like) boundary models. There seems no correlation between the magnetic moment and atomic distance and deviation. Thus, complicated variations in the magnetic moment at the twin(-like) boundaries obtained in the present work cannot be explained from the magnetovolume effect with the local average atomic distance and the average deviation from equilibrium. This is likely to be because the spacing between the twin boundaries is narrow and the lattice disorder in the relaxed twin(-like) boundary structures is complicated.

\subsection{Relation between PDOS and magnetic moment}

It is known that the rupture of cubic symmetry at the GB prevents the $t_{2 g}$-eg splitting of the $d$ bands, yielding the narrowing of the $d$ bands and an enhanced magnetic moment [1]. In twin boundaries as well, the breaking of the cubic symmetry while maintaining the same number of nearest-neighbor atoms can induce the rearrangement of the $d$ bands. Hence, the relation between the partial DOS (PDOS) and the magnetic moment were investigated on some atoms in the $f c c / f c c$, $h c p / h c p(1), h c p / h c p(2)$ and $f c c / h c p$. The PDOS of $3 d$ orbitals for spin-up and -down electrons are shown in Fig. 3 for (a) Co1 and (b) Co2 in the $f c c / f c c$, where the solid line is the PDOS for the $f c c / f c c$ 
and the dashed line is the PDOS for the bulk $f c c$. The difference in PDOS width was negligible both between Co1 and the bulk and between Co2 and the bulk. Hence, the enhanced magnetic moment for the $f c c / f c c$ cannot be explained by the narrowing of the PDOS.

The PDOS of $3 d$ orbitals for spin-up and -down electrons are shown in Fig. 4 for (a) Co1, (b) Co2 and (c) Co7 in the $h c p / h c p(1)$, where the dashed line is the PDOS for the bulk hcp. A narrowed PDOS width was found for Co1, Co2 and Co7. This trend of a narrowed PDOS width was obtained for all other atoms in the hcp/hcp(1). As shown in Table 1, an enhanced magnetic moment was found only in Co7, while the magnetic moments of Co2-Co5 were less than those of the bulk hcp, which do not correspond to a narrowed PDOS width.

The PDOS of $3 d$ orbitals for spin-up and -down electrons are shown in Fig. 5 for (a) Co1, (b) Co2 and (c) Co4 in the hcp/hcp(2). A broadened PDOS width was found in Co4, which corresponds to the reduced magnetic moment. On the other hand, there was no difference in the PDOS width between Co1 and the bulk hcp. This does not agree with the result that the magnetic moment of Co1 was less than that of the bulk hcp. In the case of Co2, the PDOS width was broadened; however, there was no difference in magnetic moment between Co2 and the bulk hcp. The PDOS of $3 d$ orbitals for spin-up and -down electrons is shown in Fig. 6 for Co7 in the $f c c / h c p$. The magnetic moment of Co7 was lower than that of the bulk hcp, but its PDOS width was almost the same as that of the bulk hcp. Thus, the variations in the magnetic moment at the $f c c / f c c, h c p / h c p$ and $f c c / h c p$ cannot be explained only by the narrowing or broadening of the PDOS width. 
Recently, it has been reported that the magnetic moment depends on the number of occupied states for the spin-down channel [4,31]. As shown in Fig. 4 (a), the number of occupied states for the spin down channel of Co1 in the $h c p / h c p(1)$ was more than that of the bulk hcp, although its PDOS width was narrowed. Therefore, it appears that the finding that the magnetic moment of Co1 in the $h c p / h c p(1)$ is equal to that of the bulk hcp results from the offset of the narrowed PDOS width and an increase in the number of occupied states for the spin-down channel. In the $h c p / h c p(2)$, the number of occupied states for the spin-down channel of Co1 was lower than that the bulk hcp, which corresponds to the enhanced moment of Co1 in the $h c p / h c p(2)$. In the case of Co2 in the $h c p / h c p(2)$, a broadened PDOS width was found, while the number of occupied states for the spin-down channel of Co1 was less than that of the bulk hcp. Therefore, the offset of these effects is responsible for the the magnetic moment of $\mathrm{Co} 2$ being equal to that of the bulk hcp. Thus, the number of occupied states for the spin-down channel state remarkably affects the magnetism of the boundaries. However, there are still some exceptions; for example, the enhanced magnetic moment of Co2 for the $f c c / f c c$ and the reduced magnetic moment of Co2 for the $h c p / h c p(1)$.

In the Stoner theory of itinerant magnetisms, the origin of a ferromagnetic order is explained by a rigid shift of the spin-up and spin-down bands under the influence of the exchange interaction. Čak et al. [3] noted that the variation in the magnetic moment at the GB can be understood by the Stoner theory of itinerant magnetisms. The PDOS of $3 d$ orbitals is shown in Fig. 7 (a) for Co2 in the $f c c / f c c$ and in Fig. 7 (b) for the Co2 in the $h c p / h c p(1)$. The inspection of Fig. 7 reveals that the value of the 
integral of the PDOS from the peak at $-1.0 \mathrm{eV}$ to the Fermi level for $\mathrm{Co} 2$ in the $f c c / f c c$ was slightly larger than that for the bulk $f c c$. This corresponds to the enhanced magnetic moment for Co2 in the $f c c / f c c$. Also, the value of the integral of the PDOS from the peak at $-0.9 \mathrm{eV}$ to the Fermi level for Co2 in the $h c p / h c p(1)$ was lower than that for the bulk hcp, which corresponds to the reduced magnetic moment for Co2 in the $h c p / h c p(1)$. Thus, the entire variation of the magnetic moment at the twin(-like) boundaries can be understood from the narrowing (or broadening) of the PDOS width of $3 d$ orbitals, the number of occupied states for the spin-down channel and the PDOS around the Fermi level.

Note that the number of $d$ electrons, which is the value of the integral of the PDOS from minus infinity to the Fermi level, for Co2 of the $h c p / h c p(1)$ was less than that of the bulk hcp. This indicates that charge transfer occurred. Moruzzi and Marcus [30] showed that charge transfer occurs when the lattice constant becomes large, resulting in an enhanced magnetic moment. Also, Takano et al. [32] showed that charge transfer plays a critical role in the enhancement of magnetic moment due to the presence of vacancies. They noted that the charge transfer gives rise to the perturbation of the Fermi level and a change in the DOS at the Fermi level. Another important effect of the charge transfer is that the numbers of s, $p$ and $d$ electrons occupied in the outermost shell change. As shown in Table 2, the $4 s$ and $4 p$ electrons give rise to diamagnetism. The contributions of $4 s$ and $4 p$ in the $h c p / h c p(1)$ were larger than those in the $f c c / f c c$. This indicates the importance of $s$ and $p$ electrons in determining magnetic properties in the case of charge transfer. 


\section{Conclusions}

The magnetic moments of the $f c c / f c c, h c p / h c p$ twin and $f c c / h c p$ twin-like boundaries in cobalt were investigated by first-principles calculations based on DFT using the CASTEP code. The magnetic moments in the $f c c / f_{c c}$ were larger than those of the bulk $f c c$. On the other hand, in the $h c p / h c p$ and $f c c / h c p$, the variations in the magnetic moment were complicated owing to the relaxation or reconstruction at the twin boundaries.

The complicated variations in the magnetic moment at the twin boundaries could not be explained by the local average atomic distance and the average deviation from equilibrium. The narrowing (or broadening) of the PDOS width of $3 d$ orbitals, the number of occupied states for the spin-down channel and the PDOS around the Fermi level were responsible for the entire variation in the magnetic moment at the twin boundaries.

In an atom in the $h c p / h c p$, charge transfer occurred. In this case, the numbers of $s, p$ and $d$ electrons occupying in the outermost shell were varied, and the contributions of $4 s$ and $4 p$ to the magnetic moment were relatively larger than those in the case of no charge transfer. 


\section{References}

[1] K. Hampel, D. D. Vvedensky: Phys. Rev. B 47, 4810 (1993).

[2] P. Bloński, A. Kiejna: Surf. Sci. 601, 123 (2007).

[3] M. Čak, M. Šob, J. Hafner: Phys. Rev. B 78, 054418 (2008).

[4] E. Wachowicz, A. Kiejna: Comput. Mater. Sci. 43, 736 (2008).

[5] E. Wachowicz, T. Ossowski, A. Kiejna: Phys. Rev. B 81, 094104 (2010).

[6] M. Tischer, O. Hjortstam, D. Arvanitis, J. H. Dunn, F. May, K. Baberschke, J. Trygg, J. M. Wills, B. Johansson, O. Eriksson: Phys. Rev. Lett. 75, 1602 (1995).

[7] M. R. Fitzsimmons, A. Röll, E. Burkel, K. E. Sickafus, M. A. Nastasi, G. S. Smith, R. Pynn: Nanostruct. Mater. 6, 539 (1995).

[8] K. Suzuki, A. Makino, A. Inoue, T. Masumoto: J. Appl. Phys. 70, 6232 (1991).

[9] M. J. Aus, B. Szpunar, A. M. El Sherik, U. Erb, G. Palumbo, K. T. Aust: Scr. Metall. 27, 1639 (1992).

[10] B. Szpunar, U. Erb, G. Palumbo, K. T. Aust, L. J. Lewis: Phys. Rev. B 53, 5547 (1996).

[11] L. Yan, Y. Wang, J. Li, A. Pyatakov, D. Viehland: J. Appl. Phys. 104, 123910 (2008).

[12] S. Li, Y. B. Zhang, W. Gao, C. Q. Sun, S. Widjaja, P. Hing: Appl. Phys. Lett. 79, 1330 (2001).

[13] B. Sampedro, P. Crespo, A. Hernando, R. Litrán, J. C. Sánchez López, C. López Cartes, A. Fernandez, J. Ramirez, J. González Calbet, M. Vallet: Phys. Rev. Lett. 91, 237203 (2003).

[14] M. A. Garcia, M. L. Ruiz-González, G. F. de la Fuente, P. Crespo, J. M. González, J. Llopis, J. 
M. González-Calbet, M. Vallet-Regi, A. Hernando: Chem. Mater. 19, 889 (2007).

[15] S. S. Alexandre, E. Anglada, J. M. Soler, F. Yndurain: Phys. Rev. B 74, 054405 (2006).

[16] M. Yuasa, H. Nakano, Y. Nakamoto, M. Hakamada, M. Mabuchi: Mater. Trans. 50, 419 (2009).

[17] H. Nakano, M. Yuasa, M. Mabuchi: Scr. Mater. 61, 371 (2009).

[18] O. Kirakami, H. Sato, Y. Shimada, F. Sato, M. Tanaka: Phys. Rev. B 56, 13849 (1997).

[19] M. D. Segall, P. J. D. Lindan, M. J. Probert, C. J. Pickard, P. J. Hasnip, S. J. Clark, M. C. Payne: J. Phys.: Condens. Matter 14, 2717 (2002).

[20] M. Tischer, O. Hjortstam, D. Arvanitis, J. H. Dunn, F. May, K. Baberschke, J. Trygg, J. M. Wills, B. Johansson, O. Eriksson: Phys. Rev. Lett. 75, 1602 (1995).

[21] D. A. Eastham, Y. Qiang, T. H. Maddock, J. Kraft, J.-P. Schille, G. S. Thompson, H. Haberland: J. Phys.: Condens. Matter 9, L497 (1997).

[22] Y. Xie, J. A. Blackman: Phys. Rev. B 66, 155417 (2002).

[23] X. Chuanyun, Y. Jinlong, D. Kaiming, W. Kelin: Phys. Rev. B 55, 3677 (1997).

[24] R. N. Nogueira, H. M. Petrilli: Phys. Rev. B 63, 012405 (2000).

[25] S. V. Dudiy, B. I. Lundqvist: Phys. Rev. B 64, 045403 (2001).

[26] S. Polesya, O. Šipr, S. Bornemann, J. Minár, H. Ebert: Europhys. Lett. 74, 1074 (2006).

[27] B. T. Naughton, D. R. Clarke: J. Am. Ceram. Soc. 90, 3541 (2007).

[28] J. Lee, G. Lauhoff, M. Tselepi, S. Hope, P. Rosenbusch, J. A. C. Bland, H. A. Dürr, G. van der Laan, J. Ph. Schillé, J. A. D. Matthew: Phys. Rev. B 55, 15103 (1997). 
[29] A. Sakuma: J. Magn. Magn. Mater. 102, 127 (1991).

[30] Y. L. Moruzzi, P. M. Marcus: Phys. Rev. B 39, 471 (1989).

[31] R. Wu, A. J. Freeman, G. B. Olson: J. Mater. Res. 7, 2403 (1992).

[32] N. Takano, T. Kai, K. Shiiki, F. Terasaki: Solid State Commun. 97, 153 (1996). 


\section{Table and Figure Captions}

Table 1 Magnetic moments of the Co atoms for the $f c c / f c c, h c p / h c p(1), h c p / h c p(2)$ and $f c c / h c p$. The location of each atom is shown in Fig. 1.

Table 2 Individual contributions of $3 d, 4 s$ and $4 p$ electrons to the magnetic moments (in $\mu$ b) for the $f c c / f c c, h c p / h c p(1), h c p / h c p(2), f c c / h c p$, bulk $f c c$ and bulk $h c p$ in cobalt. The magnetic $3 d$ electrons dominantly contribute to the magnetic moments.

Fig. 1 Four models of twin(-like) boundaries in cobalt: (a) fcc/fcc, (b) $h c p / h c p(1)$, (c) $h c p / h c p(2)$ and (d) $f c c / h c p$. The stacking sequence is ABCBACABCBAC for the $f c c / f c c$ model, and ABCABABABCABAB for the $f c c / h c p$ model along the [111] direction. The twin planes of the $h c p / h c p(1)$ and $h c p / h c p$ (2) models are (10 $\overline{1} 1) /(10 \overline{1} 1)$ and $(11 \overline{2} 4) /(11 \overline{2} 4)$, respectively.

Fig. 2 Plots of (a) the magnetic moment $v s$ the local average atomic distance and (b) the magnetic moment vs the average deviation from equilibrium for the twin(-like) models of Co.

Fig. 3 PDOS of $3 d$ orbitals for spin-up and -down electrons for (a) Co1 and (b) Co2 in the $f c c / f c c$, where the solid line is the PDOS for the $f c c / f c c$ and the dashed line is the PDOS for the bulk fCC. 
Fig. 4 PDOS of 3d orbitals for spin-up and -down electrons for (a) Co1, (b) Co2 and (c) Co7 in the $h c p / h c p(1)$, where the solid line is the PDOS for the $h c p / h c p(1)$ and the dashed line is the PDOS for the bulk hcp.

Fig. 5 PDOS of $3 d$ orbitals for spin-up and -down electrons for (a) Co1, (b) Co2 and (c) Co4 in the $h c p / h c p(2)$, where the solid line is the PDOS for the $h c p / h c p(2)$ and the dashed line is the PDOS for the bulk hcp.

Fig. 6 PDOS of $3 d$ orbitals for spin-up and -down electrons for Co7 in the $f c c / h c p$, where the solid line is the PDOS for the $f c c / h c p$ and the dashed line is the PDOS for the bulk hcp.

Fig. 7 PDOS of $3 d$ orbitals for (a) Co2 in the $f c c / f c c$ and (b) $C o 2$ in the $h c p / h c p(1)$. The value of the integral of the PDOS from the peak at $-1.0 \mathrm{eV}$ to the Fermi level for $\mathrm{Co} 2$ in the $f c c / f c c$ is larger than that for the bulk $f c c$. Also, the value of the integral of the PDOS from the peak at $-0.9 \mathrm{eV}$ to the Fermi level for Co2 in the $h c p / h c p(1)$ is lower than that for the bulk $h c p$. 


\section{Tables}

\section{Table 1}

\begin{tabular}{ccccc}
\hline Site & $f c c / f c c$ & \multicolumn{4}{c}{$h c p / h c p(1) h c p / h c p(2)$} & $f c c / h c p$ \\
\hline Co1 & 1.70 & 1.68 & 1.70 & 1.66 \\
Co2 & 1.70 & 1.64 & 1.68 & 1.68 \\
Co3 & 1.70 & 1.64 & 1.68 & 1.70 \\
Co4 & 1.70 & 1.64 & 1.58 & 1.68 \\
Co5 & & 1.66 & 1.68 & 1.66 \\
Co6 & & 1.66 & 1.68 & 1.64 \\
Co7 & & & & \\
Co8 & & 1.78 & 1.58 & 1.64 \\
Co9 & & & 1.68 & \\
\hline
\end{tabular}


Table 2

\begin{tabular}{|c|c|c|c|c|c|c|c|}
\hline \multicolumn{2}{|c|}{ Site } & \multirow{2}{*}{$\frac{\text { fcc/fcc }}{1.824}$} & \multirow{2}{*}{$\begin{array}{l}\text { hсp/hcp(1) } \\
1.846\end{array}$} & \multirow{2}{*}{$\begin{array}{l}\text { hсp/hcp(2) } \\
1.832\end{array}$} & \multirow{2}{*}{$\frac{\text { fcc/hcp }}{1.803}$} & \multirow{2}{*}{$\frac{\text { fcc Co }}{1.789}$} & \multirow{2}{*}{$\frac{\text { hср Co }}{1.808}$} \\
\hline Co1 & $3 d$ & & & & & & \\
\hline & $4 s$ & -0.032 & -0.025 & -0.0225 & -0.033 & -0.032 & -0.033 \\
\hline & $4 p$ & -0.125 & -0.136 & -0.128 & -0.128 & -0.121 & -0.127 \\
\hline \multirow[t]{3}{*}{ Co2 } & $3 d$ & 1.817 & 1.797 & 1.801 & 1.806 & & \\
\hline & $4 s$ & -0.033 & -0.032 & -0.029 & -0.034 & & \\
\hline & $4 p$ & -0.120 & -0.140 & -0.122 & -0.125 & & \\
\hline \multirow[t]{3}{*}{ Co3 } & $3 d$ & 1.817 & 1.795 & 1.812 & 1.809 & & \\
\hline & $4 s$ & -0.033 & -0.031 & -0.021 & -0.034 & & \\
\hline & $4 p$ & -0.120 & -0.129 & -0.114 & -0.123 & & \\
\hline \multirow[t]{3}{*}{$\mathrm{Co} 4$} & $3 d$ & 1.824 & 1.810 & 1.736 & 1.806 & & \\
\hline & $4 s$ & -0.032 & -0.031 & -0.035 & -0.034 & & \\
\hline & $4 p$ & -0.125 & -0.134 & -0.124 & -0.125 & & \\
\hline \multirow[t]{3}{*}{ Co5 } & $3 d$ & & 1.822 & 1.799 & 1.803 & & \\
\hline & $4 \mathrm{~s}$ & & -0.029 & -0.029 & -0.033 & & \\
\hline & $4 p$ & & -0.134 & -0.118 & -0.128 & & \\
\hline \multirow[t]{3}{*}{ Co6 } & $3 d$ & & 1.828 & 1.799 & 1.794 & & \\
\hline & $4 s$ & & -0.030 & -0.029 & -0.033 & & \\
\hline & $4 p$ & & -0.137 & -0.118 & -0.132 & & \\
\hline \multirow[t]{3}{*}{ Co7 } & $3 d$ & & 1.926 & 1.736 & 1.794 & & \\
\hline & $4 s$ & & -0.014 & -0.035 & -0.033 & & \\
\hline & $4 p$ & & -0.124 & -0.124 & -0.132 & & \\
\hline \multirow[t]{3}{*}{ Co8 } & $3 d$ & & & 1.812 & & & \\
\hline & $4 s$ & & & -0.021 & & & \\
\hline & $4 p$ & & & -0.114 & & & \\
\hline \multirow[t]{3}{*}{ Co9 } & $3 d$ & & & 1.801 & & & \\
\hline & $4 s$ & & & -0.029 & & & \\
\hline & $4 p$ & & & -0.122 & & & \\
\hline
\end{tabular}

Figures 
(a)

(b)

(c)
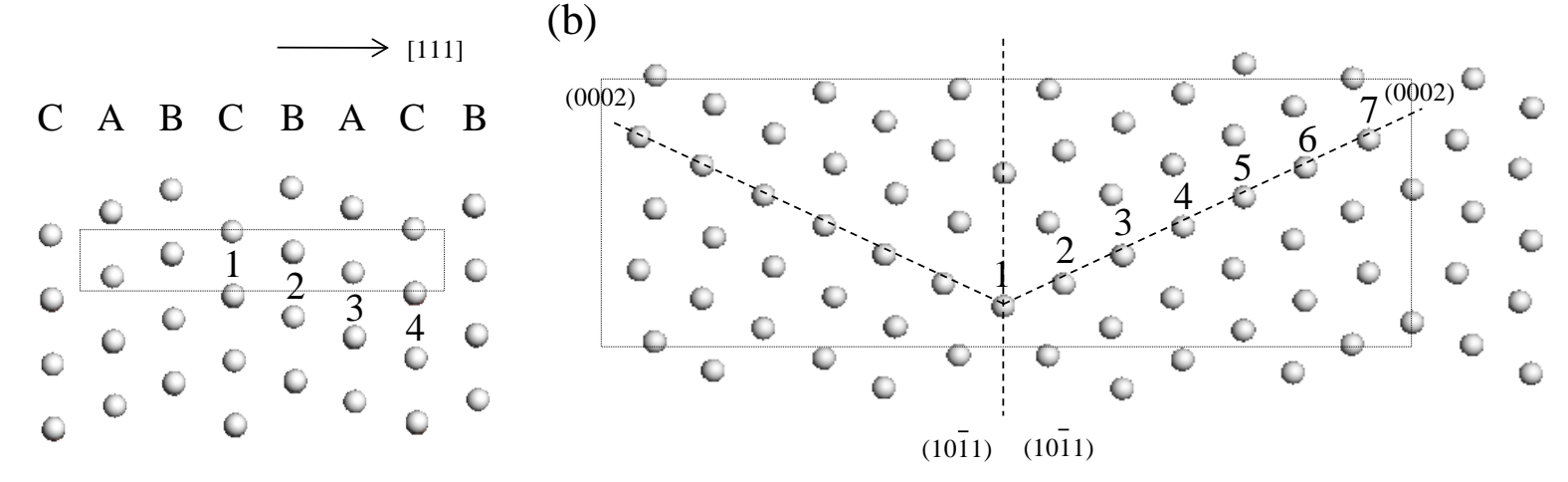

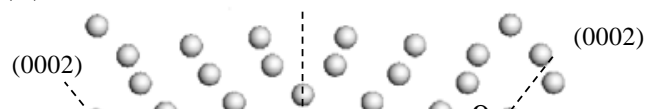

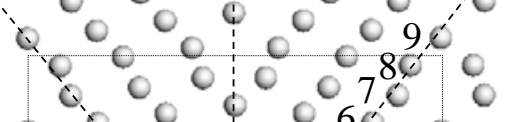

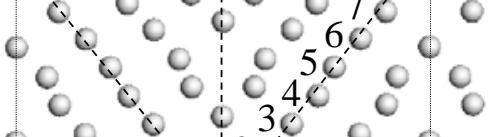

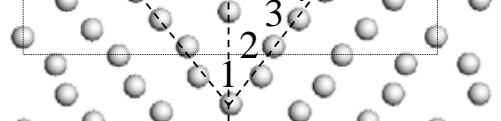

$$
\begin{aligned}
& 0.0000000 \\
& \text { (11124) (1124) }
\end{aligned}
$$

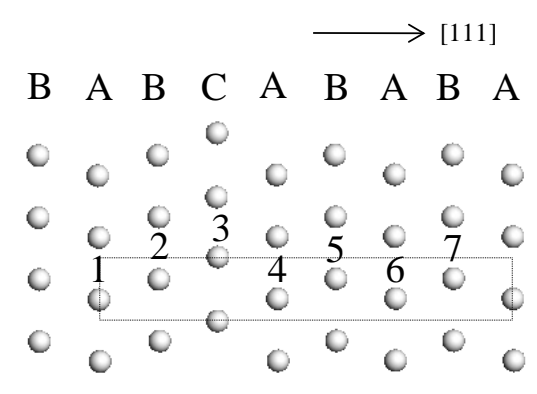

Fig. 1 
(a)

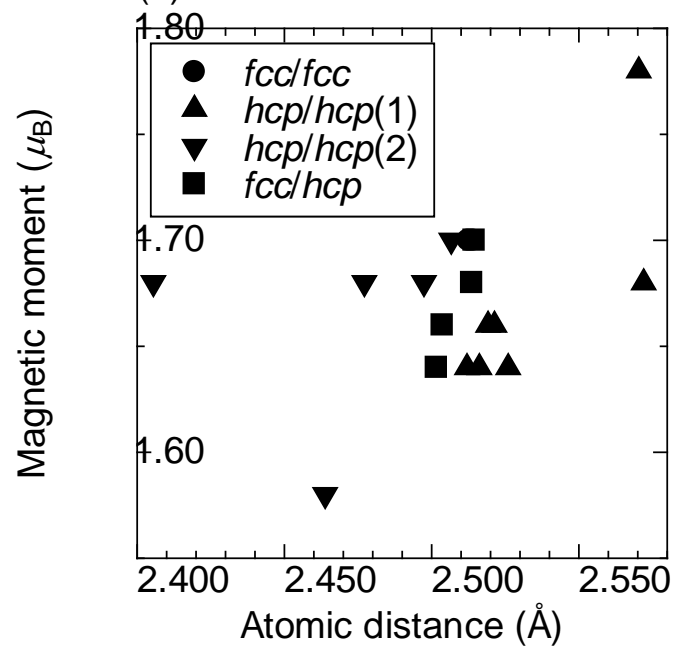

(b)

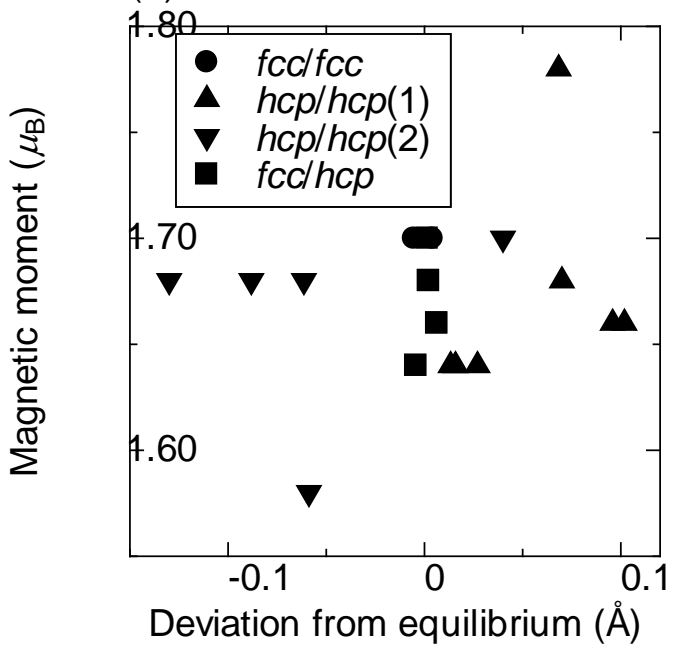

Fig. 2 

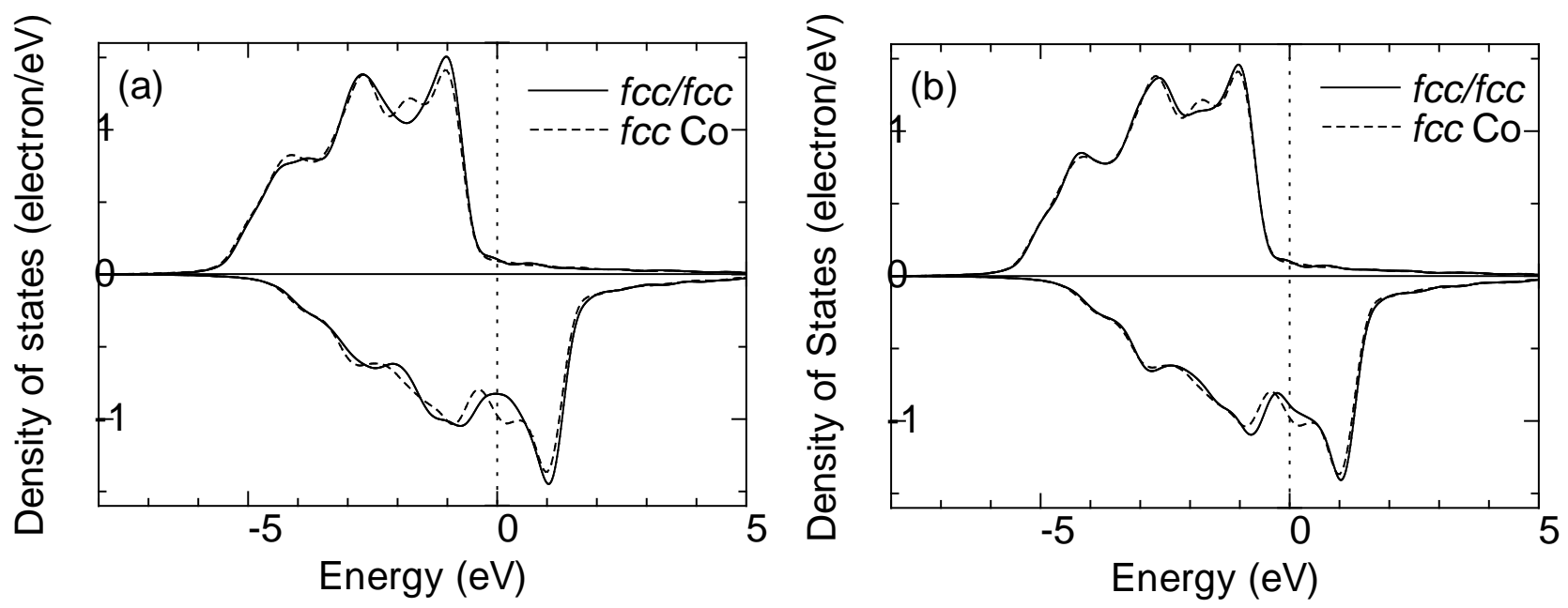

Fig. 3 

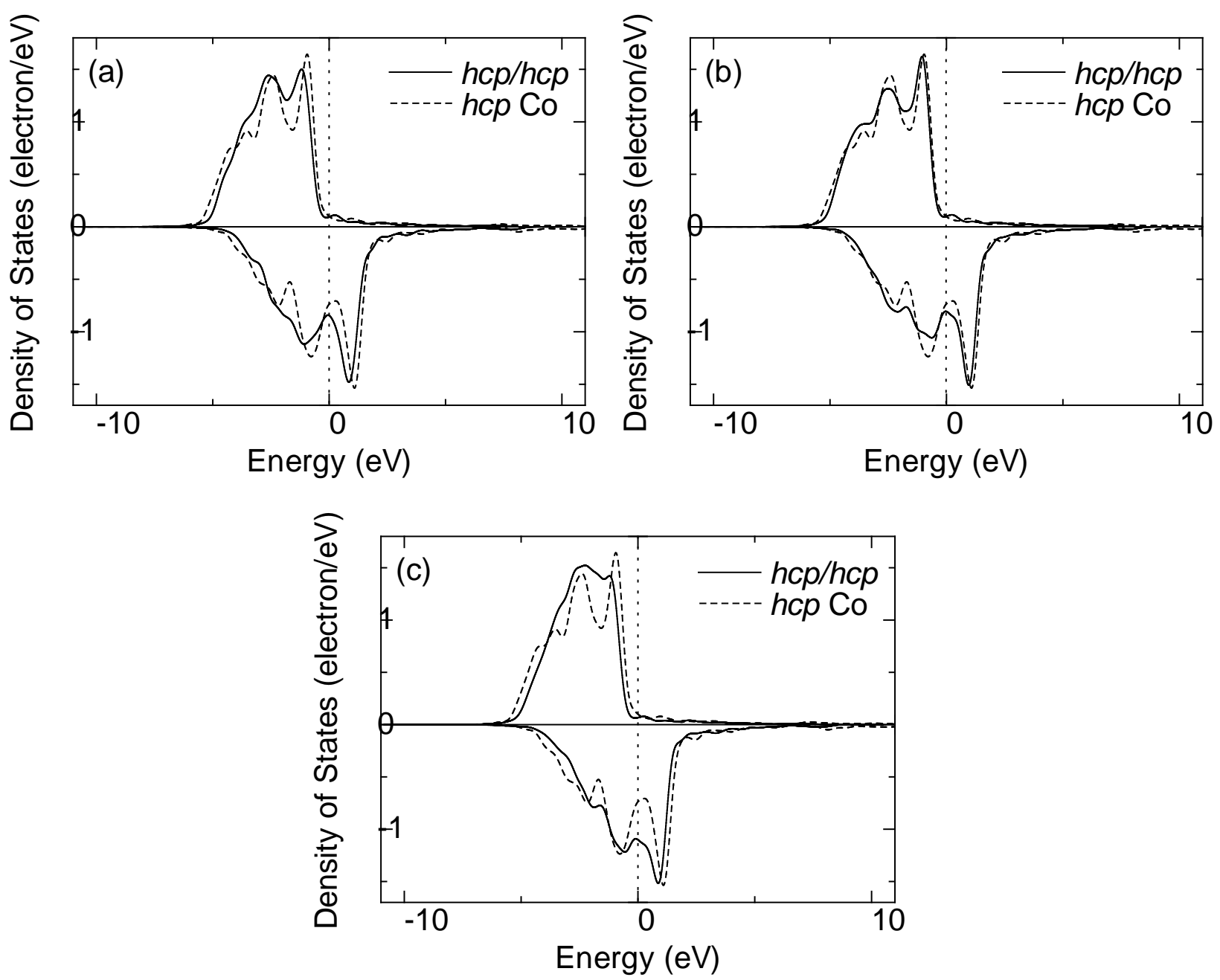

Fig. 4 

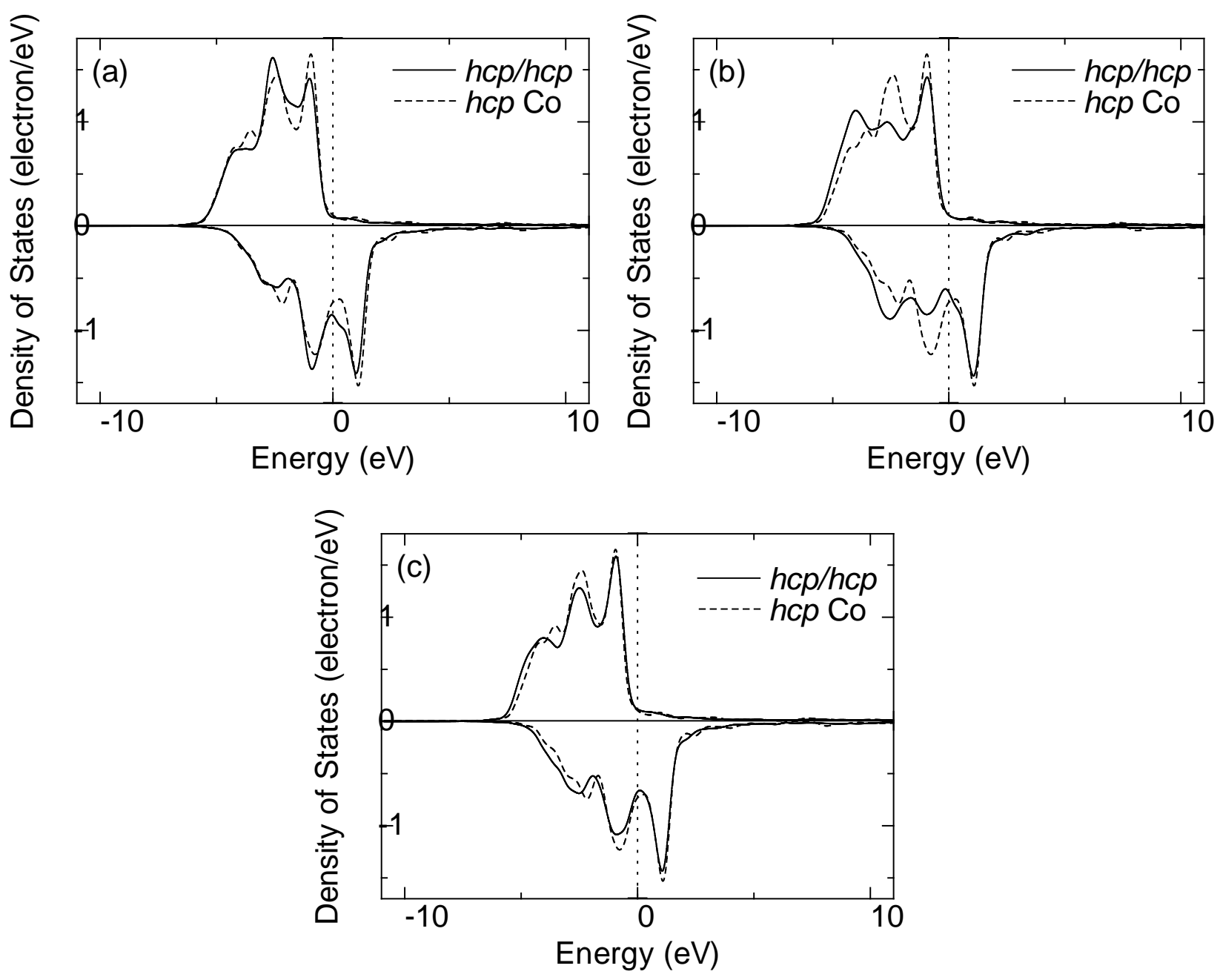

Fig. 5 


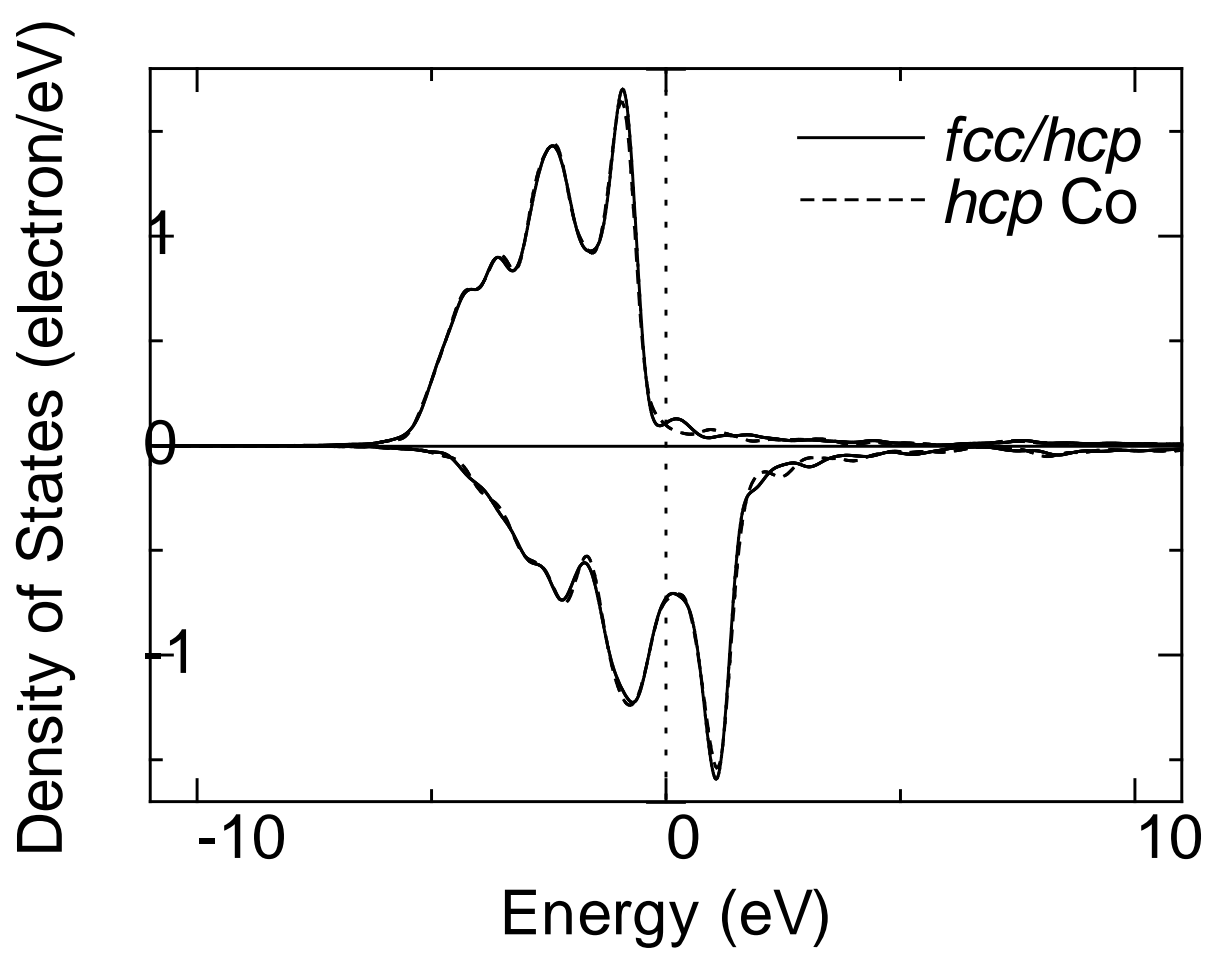

Fig. 6 

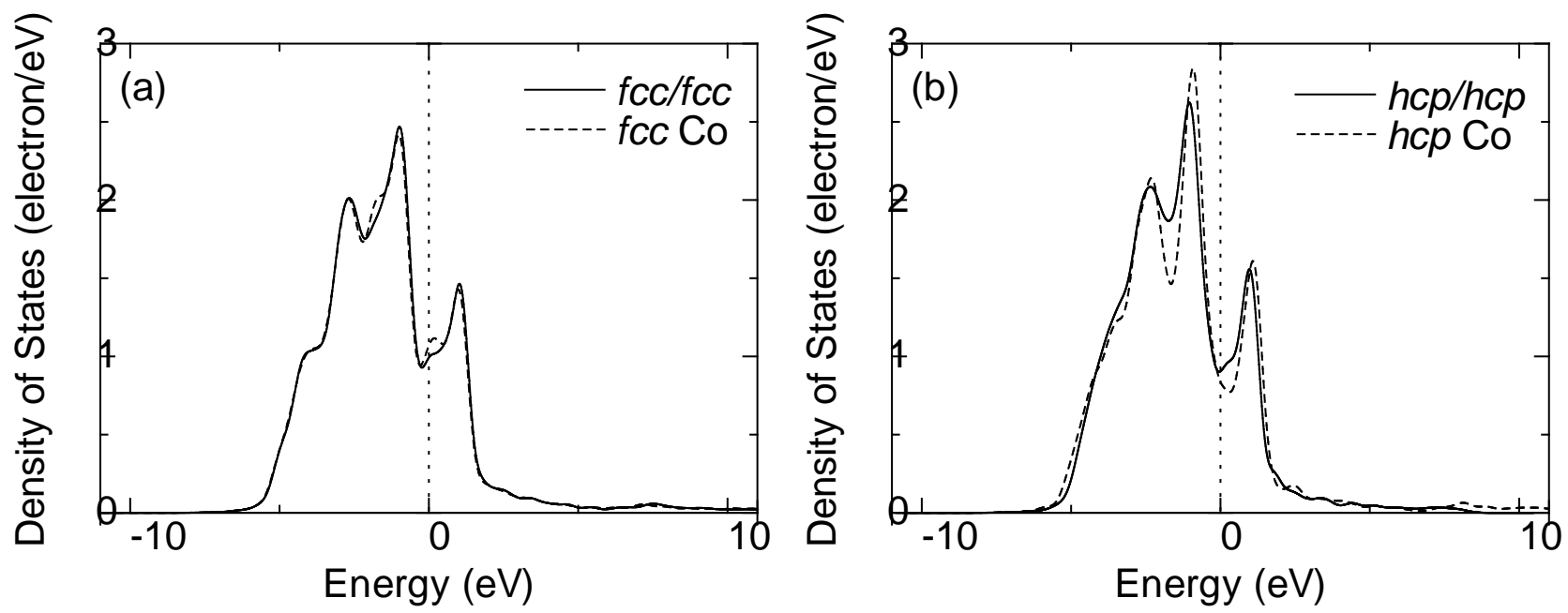

Fig. 7 